\title{
Electrochemical Micro-Machining Process Parameter Optimization Using a Neural Network-Genetic Algorithm Based Approach
}

\author{
Pan Zou, Manik Rajora, Mingyou Ma, Hungyi Chen, Wenchieh Wu, and Steven Y. Liang
}

\begin{abstract}
In spite of much work done in mapping between the process parameters and performance indicators of electrochemical micro-machining (EMM), very sparse research is available on the optimization of its process parameters. In this article, first, an ANN trained using a hybrid Simulated Annealing (SA) - Levenberg-Marquardt (LM) is developed to map between the process parameters (voltage, feed-rate, and pulse-on time) and performance indicators (inlet and outlet diameters) of EMM. Once the prediction capabilities of the ANN are verified by the use of several testing data sets, the trained ANN is then used as a fitness function to optimize the process parameters of EMM that would lead to the minimization of taper and overcut. The optimization of the process parameters was accomplished using a Genetic Algorithm (GA) based approach. The prediction model was further validates by comparing the tendencies seen in the prediction model to those obtained using partial correlation coefficient.
\end{abstract}

Index Terms-Electrochemical micro-machining (EMM), genetic algorithm (GA), levenberg-marquardt (LM) algorithm, simulated annealing (SA).

\section{INTRODUCTION}

The demand for micro-products and components has been rapidly increasing in the electronics, optics, medicine, biotechnology, automotive, communications and avionics industries [1]. Micromachining refers to the removal of material with thickness ranging from 1 to $999 \mu \mathrm{m}$ [2]. Methods include micro-electro discharge machining (micro EDM), electrochemical micro-machining (EMM), micro ultrasonic machining (micro USM), laser machining and others. In EMM, the metal workpiece is the anode, and the tool is the cathode; the electrolyte flows between the anode and cathode. A pulsed, direct current is applied to ensure localization of electrolysis dissolution. The cathode, moves several micro-meters away from the anode then discharges. According to Faraday's laws, which control the workpiece dissolution, a workpiece with desired shape or size can be obtained. EMM has many advantages over traditional material removal operations such as low tool wear, low heat,

Manuscript received September 18, 2016; revised January 17, 2017.

P. Zou is with Donghua University, Songjiang, District, Shanghai 201620, China (e-mail: zoupzp123@gmail.com).

M. Rajora and S.Y. Liang are with Georgia Institute of Technology, Atlanta, Ga, 30332, USA (e-mail: manikrajora@gmail.com, steven.liang@me.gatech.edu).

M. Ma, H. Chen, and W. Wu are with Metal Industries Research \& Development Center, Taichung 207 Taiwan, ROC (e-mail: mmy@mail.mirdc.org.tw, hyc@mail.mirdc.org.tw). and the absence of stress, however, the complexity of EMM mechanism makes the modelling between the controllable process parameters and the required performance indicators very difficult. Therefore, a suitable selection of process parameters in EMM process relies greatly on the operator's skill and experience because of the numerous possibilities of process parameters combinations. To solve this problem, researchers have designed different experimental settings [3]-[8] and tried numerous types of analysis methods, such as regression models and correlation analysis [9], [10], to map the relationship between the process parameters and the performance indicators.

The rapid development intelligent techniques and the availability of better computational capabilities bring more potential and new directions to many engineering fields. Among them, Artificial Neural Networks (ANN's) have become a widely used method for building prediction models between controllable process parameters and the required performance index. H. H. Abuzied, M. A. Awad and H. A. Senbel [11] developed a multi-layer feedforward network prediction model of resulting surface roughness and material removal rate in Electrochemical Machining (ECM) process based on the related process parameters. In the work of $\mathrm{Z}$. $\mathrm{Li}$ and H. Ji [12], an ANN was utilized to predict the machining accuracy of aero-engine blade in ECM based on five main process parameters. P. Asokan, R. Ravi Kumar, etc. [13] tested multiple regression models and ANN model, and the comparison results showed that $\mathrm{NN}$ had superior prediction capabilities compared to the regression models. Though much work has been done in the experimental investigation of the process of EMM and researchers have also developed numerous models relating the process parameters and the performance indicators, very few researchers have considered optimal process parameter selection in EMM. The selection of optimal process parameter in EMM can increase the quality of the finished product and also decrease the number of unsatisfactory products created thereby decreasing manufacturing costs and increasing customer satisfaction.

Currently, the selection of optimal process parameters is done using a blanket search method which can be extremely time-consuming. In order to overcome these issues, an ANN model is developed to model the relationship between the controllable process parameters (voltage, pulse on time and feed rate) and the performance index (internal diameter and outer diameter) based on experimental data. The training of the ANN was performed using a hybrid Simulated Annealing (SA) - Levenberg Marquardt (LM) algorithm in order to overcome the drawbacks of Gradient Descent (GD) algorithm 
that is commonly used to train the ANN. Once the prediction capabilities of the ANN were validated, it was then used to find the optimal process parameter combination that would lead to the minimization of taper and overcut.

The rest of the paper is organized in the following manner: Section II describes the experimental setup while Section III describes the ANN modeling technique and the GA based optimization approach. Section IV presents and discusses the results obtained after the training of the ANN, optimization of the process parameters, and the partial correlation analysis. Section V develops some conclusions based on the results presented in Section IV.

\section{EXPERIMENTATION}

\section{A. Experimental Setup}

Fig. 1 schematically depicts the EMM experimental setup. The system consists of a three-dimensional movement device, a small-scale power supply of $100 \mathrm{~A}$, and an electrolyte pump and filter. The feeding system is controlled by a PC-Based CNC Controller, RTX real-time windows kernel program, and a motion card that drives the linear motor precisely. A pulse generator supplies a periodic current to the experimental model. A digital oscilloscope ensures that the pulse generator produces a rectangular waveform with accurate amplitude. If the tool feed rate is excessive, the tool will contact the workpiece and cause a short circuit; thus, an oscilloscope is employed to detect any short circuits. Whenever the oscilloscope detects a short circuit, a signal is sent rapidly to the PC and the tool is extracted automatically until the measured voltage returns to the applied voltage. The micro array holes electrode module includes the multiple nozzle tool electrodes, PVC mask and tool fixture. The electrolyte is pumped to a multiple electrode cell and exits through the small nozzle in the form of a free standing jet directed towards the anode workpiece.
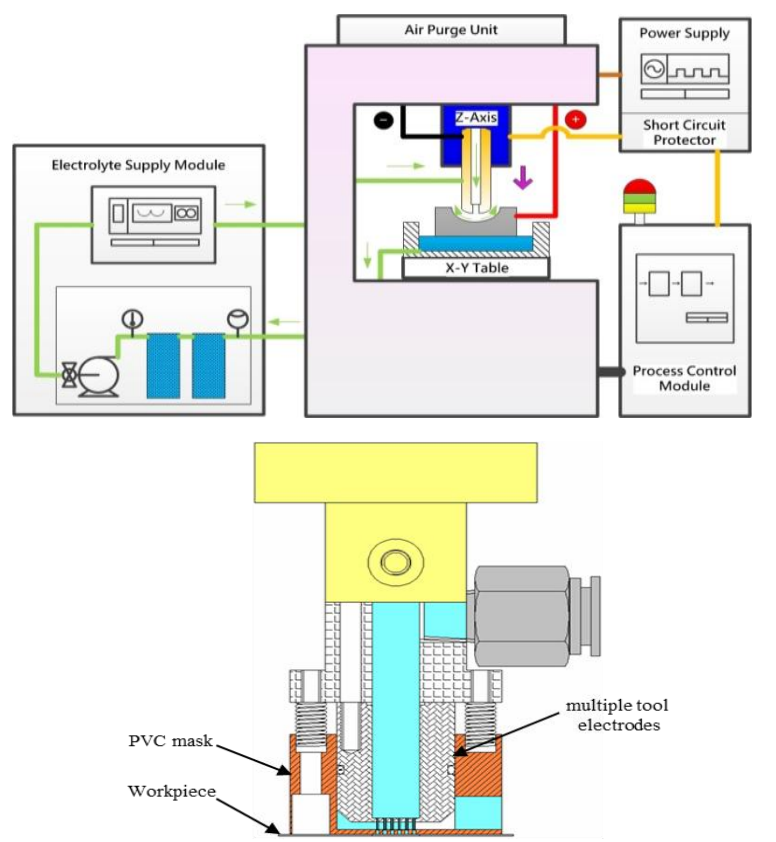

Fig. 1. Schematic diagram of electrochemical micromachining system (top) and micro array hole electrode module (bottom).

Other basic information and settings are as follows: the electrolyte velocity was $10 \mathrm{~m} / \mathrm{sec}$, electrolyte temperature was $27{ }^{\circ} \mathrm{C}$, the initial gap between the tool and the workpiece was $100 \mu \mathrm{m}$, tool moving distance was $800 \mu \mathrm{m}$, the workpiece material was SUS 304, the electrolyte used was $10 \%$ wt. $\mathrm{NaNO}_{3}$, the nominal diameter of the hole was $900 \mu \mathrm{m}$, and the depth of the hole was $500 \mu \mathrm{m}$.

Voltage, pulse on time, and feed rate were used as the controllable process parameters, while the inner diameter of the micro-hole Din and the outer diameter Dout were the measurable performances. The range of each process parameter is shown in Table I. The range of the variables was fixed by taking into consideration two factors: 1 . limitation of the devices used for EMM and 2. making sure that the experimental conditions would be stable within the chosen range. The resolution of the process parameters were was 0.1 $\mathrm{V}$ for the voltage, $0.1 \mu$ s for pulse on time, and $0.1 \mu \mathrm{m} / \mathrm{s}$ for the feed rate. This indicates that there are close to 3 million possible combinations of all the process parameters. Therefore, the proposed method was applied for this particular case study.

TABLE I: ORIGINAL RANGE OF THE CONTROLLABLE PROCESS PARAMETERS

\begin{tabular}{llll}
\hline \hline Process parameter & Voltage $(\mathrm{V})$ & $\begin{array}{l}\text { Pulse on } \\
\text { time }(\mu \mathrm{s})\end{array}$ & $\begin{array}{l}\text { Feed rate } \\
(\mu \mathrm{m} / \mathrm{s})\end{array}$ \\
\hline Lower bound & 8 & 25 & 4 \\
Upper bound & 20 & 70 & 12 \\
\hline \hline
\end{tabular}

The process of EMM has two responses i.e. taper and overcut. When drilling micro-size holes in thin metallic foils, a major requirement is for the holes to have straight walls. The straightness of the wall can be represented by the Taper and is given by:

The process of EMM has two responses i.e. taper and overcut. When drilling micro-size holes in thin metallic foils, a major requirement is for the holes to have straight walls. The straightness of the wall can be represented by the Taper and is given by:

$$
\text { Taper }=\mid\left(D_{\text {in }}-D_{\text {out }}\right) / \text { depth } \mid
$$

In critical applications, particularly in micro instruments, the straightness of a drilled hole is also very important. Overcut, as given by equation (2), is the difference between the aim holes' diameters and actual hole diameter and is a good representation of the straightness of a drilled hole. A small overcut value represents a more precise EMM process.

$$
\text { Overcut }=\left|\left(D_{\text {in }}-D\right) / 2\right|
$$

In order to create a forward prediction model for the process of EMM, three different sets of experiments were created. In the first experimental set, voltage and feed rate had 3 levels each while pulse on time was constant which resulted in a total 9 combinations of input parameters. These combinations of input experiments were used to perform the process of EMM and for each combination $D_{\text {in }}$ and $D_{\text {out }}$ were recorded. In the second and the third experimental sets voltage, pulse on time, and feed rate had 3 levels each which resulted in 27 combinations of input process parameters for both experimental sets 2 and 3. The process of EMM was performed using the combination of inputs and the Din and Dout were again recorded. The levels of voltage, pulse on 
time, and feed rate are given in Table II.

TABLE II: LeVels of Voltage, Pulse on Time, and Feed Rate Values USED FOR THE THREE EXPERIMENTAL SETS

\begin{tabular}{llll}
\hline \hline $\begin{array}{l}\text { Experiment } \\
\text { Set \# }\end{array}$ & $\begin{array}{l}\text { Levels of Voltage } \\
(\mathrm{V})\end{array}$ & $\begin{array}{l}\text { Levels Pulse } \\
\text { On Time }(\mu \mathrm{s})\end{array}$ & $\begin{array}{l}\text { Levels Feed Rate } \\
(\mu \mathrm{m} / \mathrm{s})\end{array}$ \\
\hline 1 & {$[16,18,20]$} & 25 & {$[4,6,8]$} \\
2 & {$[4,6,8]$} & {$[50,60,70]$} & {$[8,10,12]$} \\
3 & {$[4,6,8]$} & {$[50,60,70]$} & {$[8,9,10]$} \\
\hline \hline
\end{tabular}

In the experiments, Charge Coupled Device (CCD) camera was utilized to measure all the workpieces after the process of EMM. Fig. 3 shows the pictures taken with the CCD camera. The CCD images were then processed through a software which provided the average value to the diameters of the holes on the front and the backside of the workpiece.

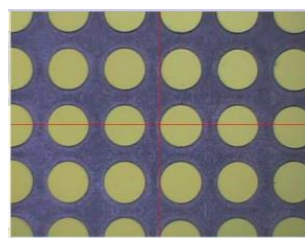

a.

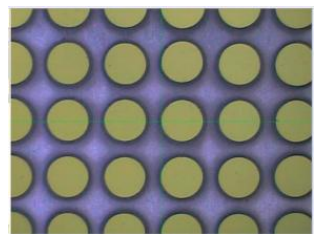

b.
Fig. 1. Pictures taken using the CCD camera. 5a. shows the front side of the workpiece while $5 \mathrm{~b}$. shows the backside of the workpiece.

\section{MODELING AND OPTIMIZATION}

\section{A. Forward Prediction Modelling}

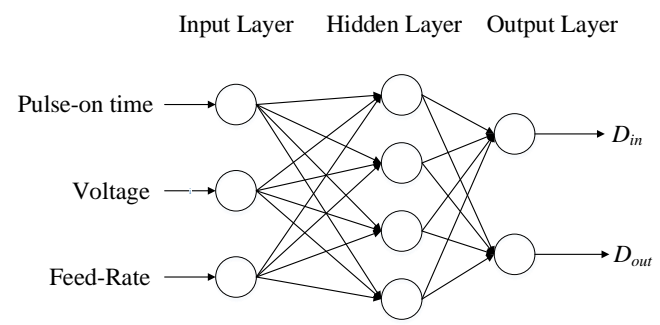

Fig. 2. Neural Network Structure used for prediction of outputs.

ANNs [14] are widely used as modeling tools in different engineering fields due to their ability to map from one multivariable space of information to another. They are able to approximating functions to the desired degree of accuracy and unlike physics based models, the shape of the approximation function does not need to be assumed before training. An ANN as seen in Fig. 2, consists of a number of input, hidden, and output neurons that interact with each other via weighted connections. The ANN's can be trained to accurately predict outputs when provided with input values by changing the values of the weighted connections. During the training process of the ANN, the ANN is provided with a set of input and outputs called the training set and the weights of the ANN are changed with aim of minimizing the mean squared error (MSE), as given by equation (3), for the training set. In this case study, the inputs of the ANN were pulse-on time, voltage, and feed-rate while the outputs were Din and Dout.

$$
M S E=\frac{1}{N} \sum_{i=1}^{N}\left(y_{i, \text { actual }}-y_{i, \text { predicted }}\right)^{2}
$$

where, $y_{i, \text { actual }}$ is the actual output and $y_{i \text {,predicted }}$ is the output predicted by the ANN.

Gradient Descent (GD) is a commonly used algorithm to train the ANN due to its ease of implementation, computational simplicity, and good results obtained for a large number of problems in different. In GD the weights are adjusted backwards, from the output layer towards the input layer. As the name implies, GD is a gradient based algorithm, therefore, depending on the starting point it might converge to the local minima instead of the global. Another drawback of GD is that it is relatively slow when close to the minima. The results obtained by gradient descent can be further improved by considering both the gradient of the error surface as well as the curvature information. In order to overcome these drawbacks, the ANN is trained using a hybrid SA-LM algorithm. Equations (4) and (5) show that GD only relies upon the gradient of the error surface. SA is a metaheuristic algorithm is able to avoid local minima and move global minimum while LM, a gradient based algorithm, a variation of gradient descent, is able to converge towards the global minimum.

\section{1) Simulated annealing}

Simulated annealing is a stochastic optimization technique for nonlinear programming that resembles the physical phenomenon of cooling metals in which atoms move from a random state to a maximally organized state. The steps of simulated annealing are outlined below [15]:

1. Initialize temperature $T$, iterations $I=0, i=1$, maximum number of points at constant $T, N$, and $M$ iteration limit.

2. Generate a random feasible point $x_{\mathrm{o}}$ and evaluate the fitness function $\mathrm{f}$ at the initial point, $f\left(x_{\mathrm{o}}\right)$.

3. Move a set distance in a random direction from the initial point and evaluate the function at the new point, $f\left(x_{1}\right)$.

4. If $f\left(x_{1}\right)<f\left(x_{0}\right)$ then $x_{1}$ becomes the new point. If $f\left(x_{1}\right)>$ $f\left(x_{0}\right)$ then $x_{1}$ is accepted as the new point based on an acceptance function given by Equation (5)

$$
P=\frac{1}{1+\exp \left(\frac{f\left(x_{1}\right)-f\left(x_{0}\right)}{T}\right)}
$$

5. If $i<N$ and the new point is accepted then $i=i+1$ otherwise reduce the temperature. If $I<M$ then $I=I+1, i=1$.

The ability to accept worse points allows the algorithm to move out of valleys containing the local minima's and search surrounding valleys for better solutions. As it can be seen from equation (6), the probability to accept worse solutions decreases as the number of iterations increases, thereby allowing the algorithm to converge towards the minima of the valley it is located in.

\section{2) Levenberg-Marquardt}

Levenberg Marquardt is a variation of GD that has the local search capabilities of Gauss Newton and the error reduction of GD algorithm and shown the ability to outperform GD in a variety of problems [16]-[18]. The weights of the ANN are updated using Equation (6) [19]

$$
\begin{gathered}
w_{k+1}=w_{k}-\left(J_{k}^{T} J_{k}+\mu I\right)^{-1} J_{k} e_{k} \\
H=J_{k}^{T} J_{k}+\mu I
\end{gathered}
$$

where, $J_{k}$ is the an approximation of the Jacobian of the MSE, 
$H$ is the Hessian of the MSE, and $\mu$ is a parameter whose value decreases when the error decrease in order to reduce the influence of the gradient and increases if the error increases. Equations (6) and (7) show that LM algorithm uses information about the gradient of the error surface and the curvature, without actually calculating the curvature, in order to update the weight values.

The number of experimental data group is 63, which means this is a small sample data analysis problem and overtraining of the ANN needs to be avoided when dealing with small sample data. Overfitting usually occurs when a model describes random error or noise instead of the underlying relationship. The potential for overfitting problem will increase when the number of training data is not far more the number of parameters, which may lead a poor predictive performance of the neural network model. Additional techniques, such as cross validation, regularization, early stopping, are very necessary with the aim of avoiding overfitting.

The data set provided were divided into training (45), validation (13), and testing (5) sets. The weights were first trained using SA with the objective of minimizing the MSE for the training set. The set of weight values from training procedure that provided the smallest MSE for the validation set were used as a starting point for LM. LM was then used to further improve these weight values with the objective of minimizing the MSE of the training set. The set of weights that provided the least MSE for the validation set were used as the final weight values for the ANN. The success of the training was measured by using the ANN to predict the outputs for the testing set and calculating the relative error between the actual output and the predicted out.

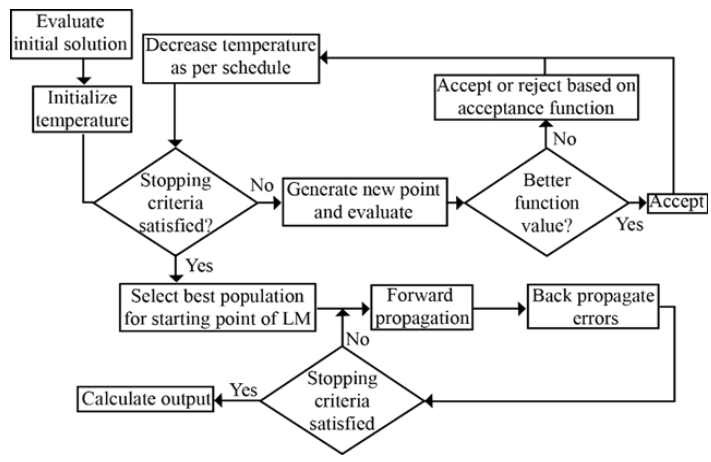

Fig. 3. Structure Flow Diagram of the Hybrid SA-LM-BP Network Training [20].

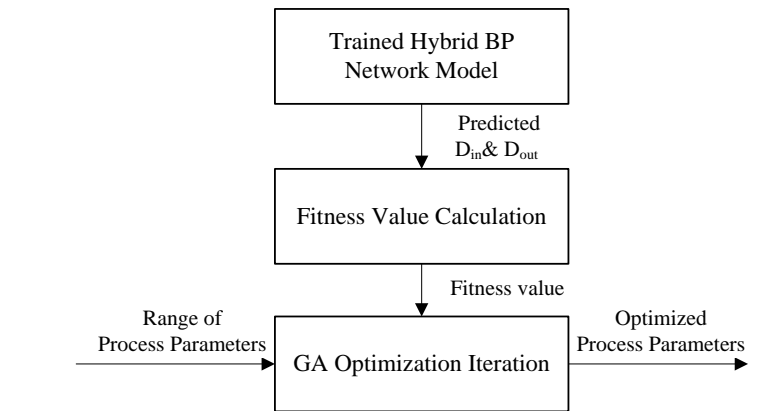

Fig. 4. Structure of the hybrid ANN-GA optimization approach in EMM process.

\section{B. ANN-GA Based Optimization Approach}

In the ANN-GA based optimization approach, ANN was used as the fitness function and GA was employed to search for the best process parameter combination that would minimize the taper and overcut. The structure of the ANN-GA optimization approach is shown in Fig. 4.

The steps of GA are outlined below:

1. Create a random initial population.

2. Create a new generation of individuals by using the current population using the following steps:

a. Calculate the fitness value of each individual of the current population

b. Select parents from these individuals based on their fitness value.

c. Select elite children, individuals with the lower fitness values that will be individuals of the next generation.

d. Produce children for the next generation from parents using either crossover or mutation.

3. Repeat step 2 until a stopping criteria is satisfied.

In the process of EMM, the aim is to find the set of process parameter combinations that will minimize both taper and overcut. Though EMM has two responses i.e. taper and overcut, for the purpose of this case study, the two responses were combined into a single-objective by the use of weight values as given by Equation (8).

$$
\text { Objective }=w_{1} \times \text { taper }+w_{2} \times \text { overcut }
$$

The weight values $w_{1}$ and $w_{2}$ can be assigned in accordance with the emphasis of each objective.

\section{RESULTS AND ANALYSIS}

A trial-and-error based approach was used to determine the best structure of the ANN that best generalized the data. The ANN structure that gave the lowest MSE for the training and the validation set was chosen as the structure to represent the relationship between the inputs and the outputs. During the training procedure of the ANN, SA was first used to train the weight values and the number of iterations for SA was 100 . Once the training using SA had been completed, LM was used to further train the ANN weight values for another 1000 iterations. The activation function used for the hidden layers was tangent sigmoid while the activation function of the output layer was just a linear function. As it can be seen from Table III, an ANN structure of 3-5-2 gave the lowest MSE for both the training and the validation set. The 3-5-2 structure also had one of the lowest mean absolute percentage error (MAPE) for the test sets. Fig. 5 shows the relative error between the predicted and the actual values obtained using the best ANN structure for each of the data points in the test set.

TABLE III: MSE OF THE TRAINING SET AND VALIDATION SET AND THE MAPE OF THE TESTING SET OBTAINED USING DIFFERENT ANN

\begin{tabular}{llll}
\multicolumn{4}{c}{ STRUCTURES } \\
\hline \hline \multirow{2}{*}{ Structure } & $\begin{array}{l}\text { MSE of } \\
\text { training set }\end{array}$ & $\begin{array}{l}\text { MSE of } \\
\text { validation set }\end{array}$ & $\begin{array}{l}\text { MAPE of } \\
\text { testing set }\end{array}$ \\
\hline $3-3-2$ & 0.15 & 0.09 & 0.24 \\
$3-4-2$ & 0.12 & 0.09 & 0.21 \\
$3-5-2$ & 0.11 & 0.08 & 0.19 \\
$3-6-2$ & 0.11 & 0.10 & 0.20 \\
$3-7-2$ & 0.12 & 0.13 & 0.21 \\
$3-3-3-2$ & 0.11 & 0.10 & 0.20 \\
$3-4-4-2$ & 0.16 & 0.15 & 0.25
\end{tabular}




\begin{tabular}{llll}
$3-5-5-2$ & 0.25 & 0.15 & 0.32 \\
$3-6-6-2$ & 0.12 & 0.11 & 0.19 \\
$3-7-7-2$ & 0.12 & 0.14 & 0.21 \\
\hline \hline
\end{tabular}

An important thing to notice is that in Fig. 5, all the relative errors are below $6 \%$ for the test sets. This information along with the fact that the MSE obtained for the training and validation sets using the best ANN structure were extremely low indicates that the hybrid SA-LM algorithm used to train the ANN was able to avoid converging to local minima's and was able to not only learn the underlying relationship in the training data set but it was also able to generalize the outputs for the test set with a high degree of accuracy.

Next, the trained ANN was used as the fitness function of GA in order to minimize the value of taper and overcut in accordance with equation (8). Table IV shows the settings of the GA algorithm and Table V shows the optimal process parameters and their corresponding taper and overcut values obtained after optimization.

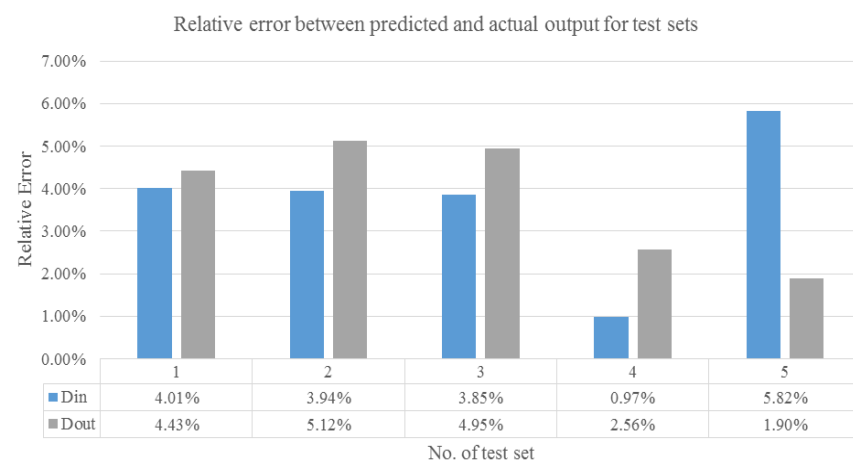

Fig. 5. Relative error between the predicted and actual outputs of the test set.

TABLE IV: PARAMETER SETTING OF HYBRID ANN-GA APPROACH

\begin{tabular}{|l|c|c|c|c|c|c|c|}
\hline $\begin{array}{l}\text { No. of hidden } \\
\text { layer }\end{array}$ & No. of neurons & Initial population & Crossover rate & Mutation rate & Elite count & $\begin{array}{c}\text { Weight value } \\
w_{1}\end{array}$ & $\begin{array}{c}\text { Weight value } \\
w_{2}\end{array}$ \\
\hline \multicolumn{1}{|c|}{4} & 20 & 0.8 & 0.1 & 1 & 0.5 & 0.5 \\
\hline \multicolumn{2}{|c|}{ Range of Voltage $(V)$} & \multicolumn{3}{|c|}{ Range of Pulse On Time $(\mu \mathrm{s})$} & \multicolumn{3}{c|}{ Range ofFeed Rate $(\mu \mathrm{m} / \mathrm{s})$} \\
\hline \multicolumn{2}{|c|}{$[8,20]$} & \multicolumn{3}{|c|}{$[4,12]$} \\
\hline
\end{tabular}

TABLE V: THE OPTIMAL RESULT OF THE HYBRID ANN-GA APPROACH

\begin{tabular}{|c|c|c|c|c|c|c|}
\hline \multicolumn{3}{|c|}{ Optimal Process Parameter } & \multicolumn{2}{c|}{ Predicted Value } & \multicolumn{2}{c|}{ Calculated Value } \\
\hline $\begin{array}{c}\text { Voltage } \\
(V)\end{array}$ & $\begin{array}{c}\text { Pulse On Time } \\
(\mu \mathrm{s})\end{array}$ & $\begin{array}{c}\text { Feed Rate } \\
(\mu \mathrm{m} / \mathrm{s})\end{array}$ & $\begin{array}{c}\text { Predicted } D_{\text {in }} \\
(\mu \mathrm{m})\end{array}$ & $\begin{array}{c}\text { Predicted } D_{\text {out }} \\
(\mu \mathrm{m})\end{array}$ & $\begin{array}{c}\text { Corresponding } \\
\text { Taper }\end{array}$ & $\begin{array}{c}\text { Corresponding Overcut } \\
(\mu \mathrm{m})\end{array}$ \\
\hline 15.4 & 37.8 & 6.9 & 902.92 & 899.78 & 0.01 & 1.46 \\
\hline
\end{tabular}

The results in Table $\mathrm{V}$ show that optimized input process parameters gave a taper and overcut value of 0.01 and 1.46 $\mu \mathrm{m}$ respectively. The taper value obtained after optimization (0.01) was very close to the best taper value available in the experimental data (0) but the optimal $D_{\text {in }}$ and $D_{\text {out }}(902.92 \mu \mathrm{m}$ and $899.78 \mu \mathrm{m}$ respectively) that gave the taper value of 0.01 were much better than the $D_{\text {in }}$ and $D_{\text {out }}(783.25 \mu \mathrm{m}$ and 783.25 $\mu \mathrm{m}$ respectively) that gave the best taper value of 0 in the experimental data. The optimal overcut value of $1.46 \mu \mathrm{m}$ was better than the best overcut value of $2 \mu \mathrm{m}$ available in the experimental data set. These results show that the optimization technique was able to find the combination of process parameters that would lead to much better performance indicators that those present in the experimental data.

\section{CONCLUSION}

In this paper, a mapping from the input parameters to the performance indicators for the process of EMM was accomplished using an ANN. In order to avoid premature convergence, a hybrid SA-LM algorithm was used to train the ANN. The 3.57\% MAPE for the testing set showed that the trained ANN was able to predict outputs for unseen data points with a very high degree of accuracy. The trained ANN was then used as a fitness function for find the optimal values of the input process parameters for the process of EMM that would lead to a small value of taper and overcut. The $D_{i n}$ and $D_{\text {out }}$ obtained after optimization were much close than any $D_{\text {in }}$ and $D_{\text {out }}$ available in the experimental data to the desired $D_{\text {in }}$ and $D_{\text {out }}$ value of $900 \mu \mathrm{m}$ and the corresponding taper (0.01) and overcut $(1.46 \mu \mathrm{m})$ were either better or quite close to the best values seen in the experimental data. The accuracy of the trained ANN model was further verified by comparing the tendencies observed in the experimental data and other literature to the tendencies of the ANN model. The results showed that the tendencies observed in the trained ANN model, the experimental data, and the literature were all very similar. These results along with the low MAPE of the testing data set prove that the ANN trained using the hybrid SA-LM algorithm was able to avoid premature convergence to local minima and was able to correctly map the relationship between the process parameters and the performance indicators. The optimization results show that GA would be a much better technique to be used in the future for optimization of process parameters instead of a blanket search method or a trial-and-error based method. The availability of the prediction and optimization model may bring more efficient and effective way to the research of EMM process without knowing much about inner mechanism of EMM itself.

Future work in this area would include development of better training techniques for ANN that would reduce the computation time and further increase the prediction accuracy. One possible ways to increase the prediction accuracy would be to combine the ANN with a physics-based model. The physics-based model would also be helpful in understanding the mechanisms of EMM and this understanding would also help with the optimization of process parameters.

\section{REFERENCES}

[1] K. P. Rajurkar, G. Levy, A. Malshe, M. M. Sundaram, J. McGeough, X. $\mathrm{Hu}, \mathrm{R}$. Resnick, and A. DeSilva, "Micro and nano machining by electro-physical and chemical processes," CIRP Annals-Manufacturing Technology, vol. 55, no. 2, pp. 643-646, Dec. 2006. 
[2] B. Bhattacharyya and J. Munda, "Experimental investigation into electrochemical micromachining (EMM) process," Journal of Materials Processing Technology, vol. 140, no. 1, pp. 287-291, Sep. 2003.

[3] M. Sen and H. S. Shan, "A review of electrochemical macro-to micro-hole drilling processes," International Journal of Machine Tools and Manufacture, vol. 45, no. 2, pp. 137-152, Feb. 2005

[4] Z. W. Fan and L. W. Hourng, "Electrochemical micro-drilling of deep holes by rotational cathode tools," The International Journal of Advanced Manufacturing Technology, vol. 52, no. 5-8, pp. 555-563, Feb. 2011.

[5] Z. W. Fan, L. W. Hourng, and M. Y. Lin, "Experimental investigation on the influence of electrochemical micro-drilling by short pulsed voltage," The International Journal of Advanced Manufacturing Technology, vol. 61, no. 9-12, pp. 957-966, Aug. 2012.

[6] W. Lei et al., "Investigation on machining accuracy of micro-holes with ultrasound disturbed electrolyte in electrochemical machining," in Proc. the 2015 International Conference on Applied Mechanics, Mechatronics and Intelligent Systems (AMMIS2015), Dec. 2015, pp. 115-120.

[7] D. Wang, Z. Zhu, J. Bao, and D. Zhu, "Reduction of stray corrosion by using iron coating in $\mathrm{NaNO}_{3}$ solution during electrochemical machining," The International Journal of Advanced Manufacturing Technology, vol. 76, no. 5-8, pp.1365-1370, Feb. 2015

[8] M. M. Lohrengel, K. P. Rataj, and T. Münninghoff, "Electrochemical Machining - mechanisms of anodic dissolution," Electrochimica Acta, vol. 201, pp. 348-353, May 2016

[9] A. Tiwari, A. Mandal, and K. Kumar, "Multi-objective optimization of electro-chemical machining by non-dominated sorting genetic algorithm," Materials Today: Proceedings, vol. 2, no. 4, pp. 2569-2575, Dec. 2015.

[10] A. Tiwari, A. Mandal, and K. Kumar, "Optimization of overcut in electrochemical machining for EN 19 tool steel using Taguchi approach," Materials Today: Proceedings, vol. 2, no. 4, pp. 2337-2345, Dec. 2015

[11] A. Sudiarso, A. A. Aladiat, and M. Mahardika, "Study on characteristics of electrochemical machining in producing multilayered microfilters," in Proc. the 1st International Conference on Science and Technology, July 2016, vol. 1755, no. 1, p. 110003

[12] H. H. Abuzied, M. A. Awad, and H. A. Senbel, "Prediction of electrochemical machining process parameters using artificial neural networks," International Journal on Computer Science and Engineering, vol. 4, no. 1, pp. 125-132, Jan. 2012.

[13] Z, Li and $\mathrm{H}$. Ji, "Machining accuracy prediction of aero-engine blade in electrochemical machining based on BP neural network," in Proc. the 2009 International Workshop on Information Security and Application, Qingdao, China, Nov. 2009.

[14] P. Asokan, R. R. Kumar, R. Jeyapaul, and M. Santhi, "Development of multi-objective optimization models for electrochemical machining process," The International Journal of Advanced Manufacturing Technology, vol. 39, no. 1-2, pp. 55-63, Oct. 2008.

[15] K. S. Narendra and K. Parthasarathy, "Identification and control of dynamical systems using neural networks," IEEE Transactions on Neural Networks, vol. 1, no. 1, pp. 4-27, Mar. 1990.

[16] H. C. Chen, J. C. Lin, Y. K. Yan, and C. H. Tsai, "Optimization of wire electrical discharge machining for pure tungsten using a neural network integrated simulated annealing approach," Expert Systems with Applications, vol. 37, no. 10, pp. 7147-7153, Oct. 2010.

[17] I. Mukherjee and S. Routroy, "Comparing the performance of neural networks developed by using Levenberg-Marquardt and Quasi-Newton with the Gradient Descent Algorithm for modelling a multiple response grinding process," Expert Systems with Applications, vol. 39, no. 3, pp. 2397-2407, Feb. 2012.

[18] O. A. Dombayc and M. Gölcü, "Daily means ambient temperature prediction using artificial neural network method: A case study of Turkey," Renewable Energy, vol. 34, no. 4, pp. 1158-1161, Apr. 2009.

[19] M. Nalbant, H. Gökkaya, I. Toktaş, and G. Sur, "Experimental investigation of the effects of uncoated, PVD- and CVD-coated cemented carbide inserts and cutting parameters on surface roughness in CNC turning and its prediction using artificial neural networks," Robotics and Computer-Integrated Manufacturing, vol. 25, no. 1, pp. 211-223, Feb. 2009.

[20] H. Yu and B. M. Wilamowski, "Levenberg-Marquardt Training," The Industrial Electronics Handbook, vol. 5, no. 12, pp. 1.

[21] J. R. Yang, W. Xu, M. Rajora, A. H. Shih, H. Z. Tan, L. W. Jin, and S. Y. Liang, "A hybrid neural network for prediction of flow shop scheduling performance," WIT Transactions on Information and Communication Technologies, vol. 67, pp. 311-318, Dec. 2014.

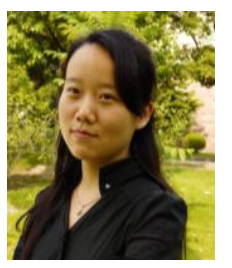

Pan Zou received her B.S. degree in mechanical engineering from Donghua University, Shanghai, China in 2012. She also received a B.S. degree in English literature from Donghua University, Shanghai, China in 2012

She is currently a $\mathrm{PhD}$ student at Donghua Univeristy. She was also a Visiting Scholar at the Georgia Institute of Technology, Atlanta, Georgia. Ms Zou's research interests include intelligent scheduling, intelligent manufacturing, and intelligent process parameter optimization.

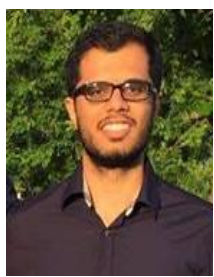

Manik Rajora received his B.S. degree from University of Louisiana at Lafayette, Louisiana, USA in mechanical engineering in 2013.

$\mathrm{He}$ is currently a Graduate Research Assistant at the Georgia Institute of Technology, Atlanta, Georgia, USA. Mr. Rajora's research interests include intelligent manufacturing, data driven decision making and intelligent process parameter optimization.

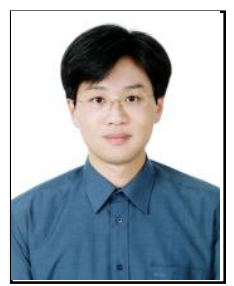

Wenchieh Wu received his B.S. degree in mechanical engineering from National Sun Yat-sen University, Kaohsiung, Taiwan, R.O.C., in 1998, and his M.S. and $\mathrm{Ph} . \mathrm{D}$. degrees in mechanical engineering from National Chiao Tung University, Hsin-Chu, Taiwan, R.O.C., in 2000 and 2005, respectively.

$\mathrm{He}$ is currently an Engineer of Section of Intelligent Technology, Metal Industries Research \& Development Centre, Taichung, Taiwan, R.O.C., where now he serves as the deputy section chief. Dr. Wu's professional interests include automatic control and system integration, servo and motion control; and customized controller development of industrial machinery. In these areas, he has published over 50 technical papers in domestic and international journals and conference proceedings. He also has over 20 Taiwan and China patents. Recent years, he is very active in carrying out scientific projects on intelligent control and monitoring of micro manufacturing.

Dr. Wu. has been invited as a thesis reviewer of National Formosa University, National Kaohsiung First University of Science and Technology; and Southern Taiwan University of Science and Technology. He also served as the Industrial-academic Cooperative Project research proposal reviewer of Ministry of Science and Technology(MOST), Taiwan, R.O.C.

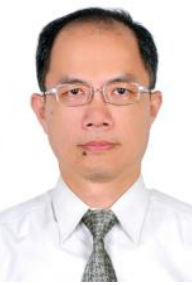

Hung-Yi Chen received the B.S. degree in mechanical engineering from Feng Chia University, Taichung, Taiwan, R.O.C., in 1989, and the M.S degrees in mechatronics engineering from National Changhua University of Education, Changhua, Taiwan, R.O.C., in 2014.

$\mathrm{He}$ is now an engineer of Section of Intelligent Technology, Metal Industries Research \& Development Centre, Taichung, Taiwan, R.O.C., where now he serves as the section chief. His professional interests include high precision machine design and electochemical machine design. He has over 27 Taiwan, China and US patents.

Currently, Dr. Chen is also the seceratary-general of Taiwan society of Electrical Machining Engineers.

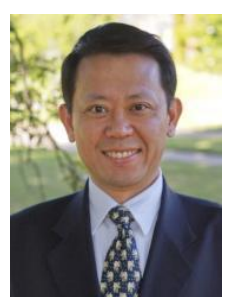

Steven Y. Liang received his B.S. degree from National Cheng-Kung University, Taiwan in 1980. He received his M.S. degree in mechanical engineering in mechanical engineering from Michigan State University in 1984 . He received his $\mathrm{PhD}$ in mechanical engineering from University of California, Berkley, California, USA in 1987.

He is currently a Morris M. Bryan Jr. Professor in mechanical engineering at the Georiga Insitute of Technology, Atlanta, USA He was the President of Walsin-Lihwa Corporatoin from 2008-2010.

Dr. Liang received the Milton C. Shaw Manufacturing Research Medal in 2016. He was also the president of the North American Manufacturing Institution (NAMRC/SME) in 2006. He also received the Robert B Doouglas Outstanding Manufacturing Engineer Award in 1991. 